\title{
Quasinormal Families of Meromorphic Functions
}

\section{Xuecheng Pang, Shahar Nevo and Lawrence Zalcman}

\begin{abstract}
Let $\mathcal{F}$ be a family of functions meromorphic on the plane domain $D$, all of whose zeros are multiple. Suppose that $f^{\prime}(z) \neq 1$ for all $f \in \mathcal{F}$ and $z \in D$. Then if $\mathcal{F}$ is quasinormal on $D$, it is quasinormal of order 1 there.
\end{abstract}

\section{Introduction}

In this paper, we are concerned with the order of quasinormality of families of meromorphic functions on plane domains, all of whose zeros are multiple.

Recall that a family $\mathcal{F}$ of functions meromorphic on a plane domain $D \subset \mathbb{C}$ is said to be quasinormal on $D$ [2] if from each sequence $\left\{f_{n}\right\} \subset \mathcal{F}$ one can extract a subsequence $\left\{f_{n_{k}}\right\}$ which converges locally uniformly with respect to the spherical metric on $D \backslash E$, where the set $E$ (which may depend on $\left\{f_{n_{k}}\right\}$ ) has no accumulation point in $D$. If $E$ can always be chosen to satisfy $|E| \leq \nu, \mathcal{F}$ is said to quasinormal of order $\nu$ on $D$. Thus a family is quasinormal of order 0 on $D$ if and only if it is normal on $D$. The family $\mathcal{F}$ is said to (quasi)normal at $z_{0} \in D$ if it is (quasi)normal on some neighborhood of $z_{0}$; thus $\mathcal{F}$ is quasinormal on $D$ if and only if it is quasinormal at each point $z \in D$. On the other hand, $\mathcal{F}$ fails to be quasinormal of order $\nu$ on $D$ precisely when there exist points $z_{1}, z_{2}, \ldots, z_{\nu+1}$ in $D$ and a sequence $\left\{f_{n}\right\} \subset \mathcal{F}$ such that no subsequence of $\left\{f_{n}\right\}$ is normal at $z_{j}, j=1,2, \ldots, \nu+1$.

Our point of departure is the following classical result of $\mathrm{Gu}$.

Theorem A ([3]). Let $\mathcal{F}$ be a family of functions meromorphic on D. If for each $f \in \mathcal{F}$ and $z \in D, f(z) \neq 0$ and $f^{\prime}(z) \neq 1$, then $\mathcal{F}$ is normal on $D$.

2000 Mathematics Subject Classification: 30D45.

Keywords: Quasinormal families, omitted values. 
Theorem A has been generalized in a number of different directions; cf., for instance, [1], [4], [7], [8]. In the present work, we are concerned with the situation in which the condition $f \neq 0$ is replaced by the assumption that all zeros of $f$ are multiple and $\mathcal{F}$ is assumed to be quasinormal on $D$. Our main result is that in this case, $\mathcal{F}$ must be quasinormal of order 1 .

Theorem. Let $\mathcal{F}$ be a quasinormal family of meromorphic functions on $D$, all of whose zeros are multiple. If for any $f \in \mathcal{F}, f^{\prime}(z) \neq 1$ for $z \in D$, then $\mathcal{F}$ is quasinormal of order 1 on $D$.

Corollary. Let $\mathcal{F}$ be a family of meromorphic functions on $D$, all of whose zeros are multiple. Suppose that each $f \in \mathcal{F}$ has at most $K$ zeros on $D$ and that $f^{\prime}(z) \neq 1$ on $D$. Then $\mathcal{F}$ is quasinormal of order 1 on $D$.

Indeed, it follows easily from Theorem $\mathrm{A}$ that $\mathcal{F}$ is quasinormal of order no greater than $K$, so the hypotheses of our Theorem are satisfied. That $\mathcal{F}$ need not be normal on $D$ is shown by the following example.

Example 1. Let $D=\{z:|z|<1\}$ and $\mathcal{F}=\left\{f_{\alpha}\right\}$, where

$$
f_{\alpha}(z)=\frac{(z+\alpha)^{2}}{z+2 \alpha}=z+\frac{\alpha^{2}}{(z+2 \alpha)}, \quad \alpha \in \mathbb{C} \backslash\{0\} .
$$

Then all zeros of $f_{\alpha}$ are multiple and $f_{\alpha}^{\prime}(z) \neq 1$. However, $f_{\alpha}$ takes on the values 0 and $\infty$ in any fixed neighborhood of 0 if $\alpha$ is sufficiently small, so $\mathcal{F}$ fails to be normal at 0 .

In certain generalizations of Gu's Theorem, the requirement that $f^{\prime}(z) \neq 1$ can be weakened to $f^{\prime}(z) \neq a(z)$, where $a(z)$ is some fixed analytic function on $D$ [4], [7], which in some cases may be required not to vanish on $D$. Unfortunately, no such extension of our theorem is available.

Example 2. Consider the family $\mathcal{F}=\left\{f_{n}\right\}$ on $D=\{z:|z|<1\}$, where

$$
f_{n}(z)=\frac{\left(z-\frac{n+2}{2 n}\right)^{2}}{z-1 / 2} .
$$

Then $\mathcal{F}$ fails to be normal at $z=1 / 2$ but is quasinormal of order 1 on $D$. Let $\varphi(z)=e^{(z+1) /(z-1)}$. Then $\varphi(D) \subset D ; \varphi^{\prime}(z) \neq 0$ on $D$; and, for each $w \in D \backslash\{0\}, \varphi^{-1}(w)$ consists of countably many points of $D$ accumulating at $z=1$. Consider the family $\tilde{\mathcal{F}}=\left\{F_{n}\right\}$ on $D$, where $F_{n}=f_{n} \circ \varphi$. Then $\tilde{\mathcal{F}}$ is a quasinormal family of meromorphic functions on $D$, all of whose zeros are multiple. Also, for any $F \in \tilde{\mathcal{F}}, \quad F^{\prime}(z)=f^{\prime}(\varphi(z)) \varphi^{\prime}(z) \neq \varphi^{\prime}(z)$ since $f^{\prime}(z) \neq 1$ for any $f \in \mathcal{F}$. However, $\tilde{\mathcal{F}}$ is not quasinormal of any finite order on $D$ as no subsequence of $\tilde{\mathcal{F}}$ is normal at any point of $\varphi^{-1}(1 / 2)$. 


\section{Notation and preliminary results}

Let us set some notation. We denote by $\Delta$ the open unit disc in $\mathbb{C}$. For $z_{0} \in \mathbb{C}$ and $r>0, \Delta\left(z_{0}, r\right)=\left\{z:\left|z-z_{0}\right|<r\right\}$ and $\Delta^{\prime}\left(z_{0}, r\right)=\left\{z: 0<\left|z-z_{0}\right|<r\right\}$. We write $f_{n} \stackrel{\chi}{\Longrightarrow} f$ on $D$ to indicate that the sequence $\left\{f_{n}\right\}$ converges to $f$ in the spherical metric uniformly on compact subsets of $D$ and $f_{n} \Longrightarrow f$ on $D$ if the convergence is in the Euclidean metric.

We require the following known results.

Lemma 1. Let $\mathcal{F}$ be a family of functions meromorphic on $\Delta$, all of whose zeros have multiplicity at least $k$, and suppose that there exists $A \geq 1$ such that $\left|f^{(k)}(z)\right| \leq A$ whenever $f(z)=0$. Then if $\mathcal{F}$ is not normal at $z_{0}$, there exist, for each $0 \leq \alpha \leq k$,

a) points $z_{n} \in \Delta, z_{n} \longrightarrow z_{0}$;

b) functions $f_{n} \in \mathcal{F}$; and

c) positive numbers $\rho_{n} \longrightarrow 0$

such that

$$
\rho_{n}^{-\alpha} f_{n}\left(z_{n}+\rho_{n} \zeta\right)=g_{n}(\zeta) \stackrel{\chi}{\Longrightarrow} g(\zeta) \text { on } \mathbb{C},
$$

where $g$ is a nonconstant meromorphic function on $\mathbb{C}$, all of whose zeros have multiplicity at least $k$, such that

$$
g^{\#}(\zeta) \leq g^{\#}(0)=k A+1 .
$$

In particular, $g$ has order at most 2 .

Here, as usual, $g^{\#}(\zeta)=\left|g^{\prime}(\zeta)\right| /\left(1+|g(\zeta)|^{2}\right)$ is the spherical derivative.

This is the local version of [6, Lemma 2] (cf. [4, Lemma 1], [9, pp. 216217]). The proof consists of a simple change of variable in the result cited from [6]; cf. [5, pp. 299-300].

Lemma 2. Let $\mathcal{F}$ be a family of functions meromorphic on $D$, all of whose zeros and poles are multiple. If for each $f \in \mathcal{F}, f^{\prime}(z) \neq 1, z \in D$, then $\mathcal{F}$ is normal on $D$.

This is the case $n=2, k=1$ of Theorem 5 in [8].

Lemma 3. Let $f$ be a nonconstant meromorphic function of finite order on $\mathbb{C}$, all of whose zeros are multiple. If $f^{\prime}(z) \neq 1$ on $\mathbb{C}$, then

$$
f(z)=\frac{(z-a)^{2}}{z-b}
$$

for some $a$ and $b(\neq a)$ in $\mathbb{C}$.

This follows from Lemma 6 (with $j=1$ and $k=2$ ) and Lemma 8 (with $k=1$ ) of [8]. 


\section{Auxiliary lemmas}

The proof of the theorem proceeds by a number of intermediate results.

Lemma 4. Let $\left\{a_{k}\right\}$ be a sequence in $\Delta$ which has no accumulation points in $\Delta$. Let $\left\{f_{n}\right\}$ be a sequence of functions meromorphic on $\Delta$, all of whose zeros are multiple, such that $f_{n}^{\prime}(z) \neq 1$ for all $n$ and all $z \in \Delta$. Suppose that

(a) no subsequence of $\left\{f_{n}\right\}$ is normal at $a_{1}$;

(b) there exists $\delta>0$ such that each $f_{n}$ has a single (multiple) zero on $\Delta\left(a_{1}, \delta\right) ;$ and

(c) $f_{n} \stackrel{\chi}{\Longrightarrow} f$ on $\Delta \backslash\left\{a_{k}\right\}_{k=1}^{\infty}$.

Then

(d) there exists $\eta_{0}>0$ such that for each $0<\eta<\eta_{0}$, $f_{n}$ has a single simple pole on $\Delta\left(a_{1}, \eta\right)$ for all sufficiently large $n$; and

(e) $f(z)=z-a_{1}$.

Proof. It suffices to prove that each subsequence of $\left\{f_{n}\right\}$ has a subsequence which satisfies (d) and (e). So suppose we have a subsequence of $\left\{f_{n}\right\}$, which (to avoid complication in notation) we again call $\left\{f_{n}\right\}$.

Since $\left\{f_{n}\right\}$ is not normal at $a_{1}$, it follows from Lemma 1 that we can extract a subsequence (which, renumbering, we continue to call $\left\{f_{n}\right\}$ ), points $z_{n} \longrightarrow a_{1}$, and positive numbers $\rho_{n} \longrightarrow 0$ such that

$$
g_{n}(\zeta)=\frac{f_{n}\left(z_{n}+\rho_{n} \zeta\right)}{\rho_{n}} \stackrel{\chi}{\Longrightarrow} g(\zeta)
$$

where $g$ is a nonconstant meromorphic function of finite order on $\mathbb{C}$, all of whose zeros are multiple. Since $g_{n}^{\prime}(\zeta)=f_{n}^{\prime}\left(z_{n}+\rho_{n} \zeta\right) \neq 1$ and $g_{n}^{\prime} \Longrightarrow g^{\prime}$ on the complement of the poles of $g$, either $g^{\prime} \neq 1$ or $g^{\prime} \equiv 1$, by Hurwitz' Theorem. In the latter case, $g(\zeta)=\zeta+c$, which does not have multiple zeros. Thus $g^{\prime}(\zeta) \neq 1$ on $\mathbb{C}$; so by Lemma 3 ,

$$
g(\zeta)=\frac{(\zeta-a)^{2}}{(\zeta-b)}
$$

for distinct complex numbers $a$ and $b$. It now follows from the argument principle that there exist sequences $\xi_{n} \longrightarrow a$ and $\eta_{n} \longrightarrow b$ such that, for sufficiently large $n, g_{n}\left(\xi_{n}\right)=0$ and $g_{n}\left(\eta_{n}\right)=\infty$. Thus, writing

$$
z_{n, 0}=z_{n}+\rho_{n} \xi_{n}, \quad z_{n, 1}=z_{n}+\rho_{n} \eta_{n},
$$

we have $z_{n, j} \longrightarrow a_{1}(j=0,1), f_{n}\left(z_{n, 0}\right)=0$ and $f_{n}\left(z_{n, 1}\right)=\infty$. 
Let us now assume that (d) has been shown to hold. It follows from Lemma 2 that the pole of $f_{n}$ at $z_{n, 1}$ is simple. The limit function $f$ from (c) is either meromorphic on $\Delta \backslash\left\{a_{k}\right\}_{k=1}^{\infty}$ or identically infinite there. Suppose first that it is meromorphic on $\Delta \backslash\left\{a_{k}\right\}_{k=1}^{\infty}$. Then there exists $\delta_{0}>0$ such that $f$ has no poles on $\Gamma=\left\{z:\left|z-a_{1}\right|=\delta_{0}\right\}$ and $f_{n}^{\prime}$ converges uniformly to $f^{\prime}$ on $\Gamma$. We claim that $f^{\prime} \equiv 1$ on $\Delta^{\prime}\left(a_{1}, \delta_{0}\right)$. Indeed, otherwise by Hurwitz' Theorem, $f^{\prime} \neq 1$. Now $1 /\left(f_{n}^{\prime}-1\right)$ is analytic on $\Delta\left(a_{1}, \delta_{0}\right)$ and converges uniformly on $\Gamma$ to $1 /\left(f^{\prime}-1\right)$. By the maximum principle, $1 /\left(f_{n}^{\prime}-1\right)$ converges uniformly on $\Delta\left(a_{1}, \delta_{0}\right)$, so $\left\{f_{n}^{\prime}\right\}$ is normal at $a_{1}$. However, since $f_{n}^{\prime}\left(z_{n, 0}\right)=0$ and $f_{n}^{\prime}\left(z_{n, 1}\right)=\infty$ and $z_{n, j} \longrightarrow a_{1}(j=0,1),\left\{f_{n}^{\prime}\right\}$ is not equicontinuous at $a_{1}$, a contradiction.

Thus $f$ has no poles on $\Delta^{\prime}\left(a_{1}, \delta_{0}\right)$ and $f_{n}^{\prime} \Longrightarrow 1$ on $\Delta^{\prime}\left(a_{1}, \delta_{0}\right)$. Hence for any $z, z_{0} \in \Delta^{\prime}\left(a_{1}, \delta_{0}\right)$

$$
f_{n}(z)-f_{n}\left(z_{0}\right)=\int_{z_{0}}^{z} f_{n}^{\prime}(\zeta) d \zeta \longrightarrow z-z_{0} .
$$

Taking a subsequence if necessary, we may suppose that $f_{n}\left(z_{0}\right)-z_{0} \longrightarrow \alpha$. We claim that $\alpha=-a_{1}$. For otherwise, taking $r<\min \left\{\left|\alpha+a_{1}\right|, \delta_{0}\right\}$, we have, for large $n$,

$$
\frac{1}{2 \pi i} \int_{\left|z-a_{1}\right|=r} \frac{f_{n}^{\prime}(z)}{f_{n}(z)} d z=\frac{1}{2 \pi i} \int_{\left|z-a_{1}\right|=r} \frac{d z}{z-a_{1}+\left(f_{n}\left(z_{0}\right)-z_{0}+a_{1}\right)}=0 .
$$

However, by the argument principle, the left hand side is the number of zeros minus the number of poles (counting multiplicities) of $f_{n}$ in $\Delta\left(a_{1}, r\right)$, which for large $n$ is at least $2-1=1$. It follows that $f(z)=z-a_{1}$.

Suppose now that $f \equiv \infty$ on $\Delta \backslash\left\{a_{k}\right\}_{k=1}^{\infty}$. Let

$$
F_{n}(z)=f_{n}(z) \frac{z-z_{n, 1}}{\left(z-z_{n, 0}\right)^{2}}
$$

By (b), $F_{n}(z) \neq 0$ on $\Delta\left(a_{1}, \delta\right)$. Applying the maximum principle to the sequence $\left\{1 / F_{n}\right\}$ of analytic functions, we see that $F_{n} \Longrightarrow \infty$ on $\Delta\left(a_{1}, \delta\right)$.

We have

$$
\begin{aligned}
\frac{f_{n}\left(z_{n}+\rho_{n} \zeta\right)}{\rho_{n}} & =\frac{F_{n}\left(z_{n}+\rho_{n} \zeta\right)}{\rho_{n}} \frac{\left(\rho_{n} \zeta+z_{n}-z_{n, 0}\right)^{2}}{\left(\rho_{n} \zeta+z_{n}-z_{n, 1}\right)} \\
& =F_{n}\left(z_{n}+\rho_{n} \zeta\right) \frac{\left(\zeta-\xi_{n}\right)^{2}}{\zeta-\eta_{n}}
\end{aligned}
$$

It follows from (3.1), (3.2) and (3.3) that $F_{n}\left(z_{n}+\rho_{n} \zeta\right) \longrightarrow 1$, which contra$\operatorname{dicts} F_{n} \Longrightarrow \infty$ near $a_{1}$. Thus the possibility $f \equiv \infty$ may be ruled out. 
We have shown that when (d) obtains, (e) does as well. Now let us show that (d) must hold. Suppose not. Then, taking a subsequence and renumbering, we may assume that on any neighborhood of $a_{1}, f_{n}$ has at least two poles for sufficiently large $n$. Keeping the notation established above, let $z_{n, 2} \neq z_{n, 1}$ be such that $f_{n}\left(z_{n, 2}\right)=\infty$ and $f_{n}$ has no poles in $\Delta^{\prime}\left(z_{n, 1},\left|z_{n, 1}-z_{n, 2}\right|\right)$. Write $z_{n, 2}=z_{n}+\rho_{n} \eta_{n}^{*}$. Then $z_{n, 2} \longrightarrow a_{1}$ but $\eta_{n}^{*} \longrightarrow \infty$ since the right hand side of (3.2) has but a single simple pole. Set

$$
G_{n}(\zeta)=\frac{f_{n}\left(z_{n, 1}+\left(z_{n, 2}-z_{n, 1}\right) \zeta\right)}{z_{n, 2}-z_{n, 1}}
$$

Since $z_{n, 2}-z_{n, 1} \longrightarrow 0, G_{n}(\zeta)$ is defined for any $\zeta \in \mathbb{C}$ if $n$ is sufficiently large; and $G_{n}^{\prime}(\zeta) \neq 1$. Now

$$
G_{n}(0)=\infty \quad G_{n}\left(\frac{z_{n, 0}-z_{n, 1}}{z_{n, 2}-z_{n, 1}}\right)=0
$$

and

$$
\frac{z_{n, 0}-z_{n, 1}}{z_{n, 2}-z_{n, 1}}=\frac{\xi_{n}-\eta_{n}}{\eta_{n}^{*}-\eta_{n}} \longrightarrow 0,
$$

so $\left\{G_{n}\right\}$ is not normal at 0 .

On the other hand, for $n$ sufficiently large, $G_{n}$ has only a single zero (which tends to 0 as $n \longrightarrow \infty$ ) on any compact subset of $\mathbb{C}$. Since $G_{n}^{\prime}(\zeta) \neq 1$, it follows from Theorem A that $\left\{G_{n}\right\}$ is normal on $\mathbb{C} \backslash\{0\}$. Taking a subsequence and renumbering, we may assume that $G_{n} \stackrel{\chi}{\Longrightarrow} G$ on $\mathbb{C} \backslash\{0\}$. Since $G$ has only a single pole on $\Delta$, conditions (a), (b), (c), and (d) hold for the sequence $\left\{G_{n}\right\}$ (defined, say, on $\left.\Delta(0,2)\right)$ with $a_{1}=0$ and $\delta=1$. Thus, by the first part of the proof, $G(\zeta)=\zeta$. But this contradicts $G(1)=\infty$. This completes the proof of Lemma 4 .

Definition. Let $z_{1}, z_{2} \in \mathbb{C}$ and put $\tilde{z}=\left(z_{1}+z_{2}\right) / 2$. We say that $\left(z_{1}, z_{2}\right)$ is a nontrivial pair of zeros of $f$ if

(i) $f\left(z_{1}\right)=f\left(z_{2}\right)=0$ and

(ii) there exists $z_{3}$ such that $\left|z_{3}-\tilde{z}\right|<\left|z_{1}-z_{2}\right|$ and $\left|f^{\prime}\left(z_{3}\right)\right|>1$.

Note that (ii) is equivalent to

(ii') there exists $z^{*}$ such that $\left|z^{*}\right|<1$ and $\left|h^{\prime}\left(z^{*}\right)\right|>1$, where

$$
h(z)=\frac{f\left(\tilde{z}+\left(z_{1}-z_{2}\right) z\right)}{z_{1}-z_{2}} .
$$

Since $\left|h^{\prime}(z)\right| \geq h^{\#}(z)$, it suffices to have $h^{\#}\left(z^{*}\right)>1$ in $\left(\mathrm{ii}^{\prime}\right)$. 
Our next result deals with the situation in which the functions $f_{n}$ have more than a single zero in each neighborhood of a point of non-normality.

Lemma 5. Let $\left\{f_{n}\right\}$ be a sequence of functions meromorphic on $\Delta$, all of whose zeros are multiple, such that $f_{n}^{\prime}(z) \neq 1$ for all $n$ and all $z \in \Delta$. Suppose that

(a) no subsequence of $\left\{f_{n}\right\}$ is normal at $z_{0}$, and

(b) for each $\delta>0, f_{n}$ has at least two distinct zeros on $\Delta\left(z_{0}, \delta\right)$ for sufficiently large $n$.

Then for each $\delta>0, f_{n}$ has a nontrivial pair $\left(a_{n}, c_{n}\right)$ of zeros on $\Delta\left(z_{0}, \delta\right)$ for sufficiently large $n$, and

$$
\left\{\frac{f_{n}\left(d_{n}+\left(a_{n}-c_{n}\right) \zeta\right)}{a_{n}-c_{n}}\right\}
$$

is not normal on $\Delta$. Here $d_{n}=\left(a_{n}+c_{n}\right) / 2$.

Proof. As in the proof of the previous lemma, it follows from (a) and Lemmas 1 and 3 that for each subsequence of $\left\{f_{n}\right\}$ there exists a (sub)subsequence (which, renumbering, we continue to denote by $\left\{f_{n}\right\}$ ), points $z_{n} \rightarrow z_{0}$, numbers $\rho_{n} \rightarrow 0^{+}$, and distinct $a, b \in \mathbb{C}$ such that

$$
g_{n}(\zeta)=\frac{f_{n}\left(z_{n}+\rho_{n} \zeta\right)}{\rho_{n}} \stackrel{\chi}{\Longrightarrow} g(\zeta)=\frac{(\zeta-a)^{2}}{\zeta-b} \quad \text { on } \quad \mathbb{C} .
$$

Thus there exist $\xi_{n} \longrightarrow a, \eta_{n} \longrightarrow b$ so that $a_{n}=z_{n}+\rho_{n} \xi_{n} \longrightarrow z_{0}, b_{n}=$ $z_{n}+\rho_{n} \eta_{n} \longrightarrow z_{0}$ and $g_{n}\left(\xi_{n}\right)=f_{n}\left(a_{n}\right)=0, g_{n}\left(\eta_{n}\right)=f_{n}\left(b_{n}\right)=\infty$ for $n$ sufficiently large.

By assumption, there also exists $c_{n} \neq a_{n}, c_{n} \longrightarrow z_{0}$, such that $f_{n}\left(c_{n}\right)=0$. Thus $c_{n}=z_{n}+\rho_{n} \xi_{n}^{*}$ and $\xi_{n}^{*} \longrightarrow \infty$ by (3.4). Setting $d_{n}=\left(a_{n}+c_{n}\right) / 2$, we see that the function

$$
h_{n}(\zeta)=\frac{f_{n}\left(d_{n}+\left(a_{n}-c_{n}\right) \zeta\right)}{a_{n}-c_{n}}
$$

is defined for any $\zeta \in \mathbb{C}$ if $n$ is sufficiently large.

We claim that $\left\{h_{n}\right\}$ is not normal at $\zeta=1 / 2$. Indeed, we have

$$
\begin{array}{cl}
\frac{a_{n}-d_{n}}{a_{n}-c_{n}} \longrightarrow \frac{1}{2}, & \frac{b_{n}-d_{n}}{a_{n}-c_{n}} \longrightarrow \frac{1}{2}, \\
h_{n}\left(\frac{a_{n}-d_{n}}{a_{n}-c_{n}}\right)=f_{n}\left(a_{n}\right)=0, & h_{n}\left(\frac{b_{n}-d_{n}}{a_{n}-c_{n}}\right)=f_{n}\left(b_{n}\right)=\infty,
\end{array}
$$

so $\left\{h_{n}\right\}$ fails to be equicontinuous in a neighborhood of $1 / 2$. 
It follows from Marty's Theorem that

$$
\lim _{n \longrightarrow \infty} \sup _{\left|\zeta-\frac{1}{2}\right| \leq \frac{1}{4}} h_{n}^{\#}(\zeta)=\infty
$$

Thus $\left(a_{n}, c_{n}\right)$ is a nontrivial pair of zeros of $f_{n}$ for $n$ sufficiently large.

Lemma 6. Let $\left\{f_{n}\right\}$ be a sequence of functions meromorphic on $\Delta$, all of whose zeros are multiple, such that $f_{n}^{\prime}(z) \neq 1$ for all $n$ and all $z \in \Delta$. Suppose that

(a) there exist $d \in \Delta, a_{n} \longrightarrow d, c_{n} \longrightarrow d$, and $z_{0} \in \mathbb{C}$ such that for every $\delta>0$,

$$
h_{n}(z)=\frac{f_{n}\left(d_{n}+\left(a_{n}-c_{n}\right) z\right)}{a_{n}-c_{n}}
$$

has at least two distinct zeros on $\Delta\left(z_{0}, \delta\right)$ for sufficiently large $n$, where $d_{n}=\left(a_{n}+c_{n}\right) / 2 ;$ and

(b) no subsequence of $\left\{h_{n}\right\}$ is normal at $z_{0}$.

Then for $n$ sufficiently large, $f_{n}$ has a nontrivial pair of zeros $\left(z_{n, 1}^{*}, z_{n, 2}^{*}\right)$ such that $z_{n, j}^{*} \longrightarrow d(j=1,2)$ and $\left|z_{n, 1}^{*}-z_{n, 2}^{*}\right|<\left|a_{n}-c_{n}\right|$.

Proof. As before, it follows from Lemmas 1 and 3 that to each subsequence of $\left\{h_{n}\right\}$ there corresponds a subsequence (which we continue to write as $\left.\left\{h_{n}\right\}\right), z_{n} \longrightarrow z_{0}$, and $\rho_{n} \longrightarrow 0^{+}$such that

$$
g_{n}(\zeta)=\frac{h_{n}\left(z_{n}+\rho_{n} \zeta\right)}{\rho_{n}} \stackrel{\chi}{\Longrightarrow} \frac{(\zeta-a)^{2}}{\zeta-b} \text { on } \mathbb{C} .
$$

Thus there exist $\xi_{n, 0} \longrightarrow b, \xi_{n, 1} \longrightarrow a$ so that

$$
z_{n, j}=z_{n}+\rho_{n} \xi_{n, j} \longrightarrow z_{0} \quad(j=0,1)
$$

and $g_{n}\left(\xi_{n, 0}\right)=h_{n}\left(z_{n, 0}\right)=\infty, g_{n}\left(\xi_{n, 1}\right)=h_{n}\left(z_{n, 1}\right)=0$. By (a), there exist $z_{n, 2} \longrightarrow z_{0}, z_{n, 2} \neq z_{n, 1}$, such that $h_{n}\left(z_{n, 2}\right)=0$. Setting $z_{n, 2}=z_{n}+\rho_{n} \xi_{n, 2}$, we have $\xi_{n, 2} \longrightarrow \infty$.

Now put

$$
z_{n, j}^{*}=d_{n}+\left(a_{n}-c_{n}\right) z_{n}+\rho_{n}\left(a_{n}-c_{n}\right) \xi_{n, j} \quad j=0,1,2 .
$$

Clearly $z_{n, j}^{*} \longrightarrow d, j=0,1,2$. Define

$$
G_{n}(\zeta)=\frac{f_{n}\left(\frac{z_{n, 1}^{*}+z_{n, 2}^{*}}{2}+\left(z_{n, 1}^{*}-z_{n, 2}^{*}\right) \zeta\right)}{z_{n, 1}^{*}-z_{n, 2}^{*}}
$$


Then $\left\{G_{n}\right\}$ is not normal at $\zeta=1 / 2$. Indeed,

$$
G_{n}\left(\frac{2 \xi_{n, 0}-\xi_{n, 1}-\xi_{n, 2}}{2\left(\xi_{n, 1}-\xi_{n, 2}\right)}\right)=\infty, \quad G_{n}(1 / 2)=0
$$

Since

$$
\frac{2 \xi_{n, 0}-\xi_{n, 1}-\xi_{n, 2}}{2\left(\xi_{n, 1}-\xi_{n, 2}\right)} \longrightarrow 1 / 2
$$

$\left\{G_{n}\right\}$ is not equicontinuous at $\zeta=1 / 2$. As before, it follows from Marty's Theorem that $\left(z_{n, 1}^{*}, z_{n, 2}^{*}\right)$ is a nontrivial pair of zeros of $f_{n}$. Now

$$
\left|z_{n, 1}^{*}-z_{n, 2}^{*}\right|=\left|a_{n}-c_{n}\right|\left|z_{n, 1}-z_{n, 2}\right|
$$

therefore, since $z_{n, j} \longrightarrow z_{0} \quad(j=1,2)$, we have $\left|z_{n, 1}^{*}-z_{n, 2}^{*}\right|<\left|a_{n}-c_{n}\right|$ for large enough $n$, as required.

Lemma 7. Let $\left\{f_{n}\right\}$ be a sequence of functions meromorphic on $\Delta$, all of whose zeros are multiple, such that $f_{n}^{\prime}(z) \neq 1$ for all $n$ and all $z \in \Delta$. Suppose that

(a) $\left\{f_{n}\right\}$ is normal on $\Delta^{\prime}(0,1)$, but no subsequence of $\left\{f_{n}\right\}$ is normal at 0 ;

(b) there exists $\delta>0$ such that $f_{n}$ has a single (multiple) zero on $\Delta(0, \delta)$ for all sufficiently large $n$.

Then there exists a subsequence of $\left\{f_{n}\right\}$ (which we continue to call $\left\{f_{n}\right\}$ ) such that for any $a \in \mathbb{C}, f_{n}-a$ has at most two zeros (counting multiplicity) on $\Delta(0,1 / 2)$.

Proof. Taking a subsequence and renumbering, we may assume that

$$
f_{n} \stackrel{\chi}{\Longrightarrow} f \text { on } \Delta^{\prime}(0,1) \text {. }
$$

By Lemma $4, f(z)=z$. Suppose that $|a| \leq 2 / 3$. Taking $\Gamma$ to be the circle $\{|z|=3 / 4\}$ traversed once in the positive direction, we have

$$
\frac{1}{2 \pi i} \int_{\Gamma} \frac{f_{n}^{\prime}(z)}{f_{n}(z)-a} d z \longrightarrow \frac{1}{2 \pi i} \int_{\Gamma} \frac{1}{z-a} d z=1
$$

However, the left hand side is the number of $a$-points of $f_{n}$ minus the number of poles of $f_{n}$ inside $\Gamma$, counting multiplicities. By Lemma 4 , there exists $0<\delta<3 / 4$ such that $f_{n}$ has a single simple pole on $\Delta(0, \delta)$ for $n$ sufficiently large.

Since $f_{n}$ converges uniformly to $z$ on $\{z: \delta \leq|z| \leq 3 / 4\}$, there exists $N_{1}$ such that if $n \geq N_{1} f_{n}$ has a single simple pole in $\Delta(0,3 / 4)$. Hence for $n \geq N_{1}, f_{n}$ takes on the value $a$ (counting multiplicities) exactly twice on $\Delta(0,3 / 4)$. 
Suppose now that $|a|>2 / 3$. Let $\Gamma^{\prime}$ be the circle $\{|z|=5 / 9\}$ traversed in the positive direction. Then

$$
\frac{1}{2 \pi i} \int_{\Gamma^{\prime}} \frac{f_{n}^{\prime}(z)}{f_{n}(z)-a} d z \longrightarrow \frac{1}{2 \pi i} \int_{\Gamma^{\prime}} \frac{1}{z-a}=0,
$$

so the number of $a$-points minus the number of poles of $f_{n}$ (counting multiplicity) inside $\Gamma^{\prime}$ is 0 for large $n$. It follows as before that there exists $N_{2}$ such that $f_{n}$ takes on the value $a$ exactly once (counting multiplicities) on $\Delta(0,5 / 9)$ if $n \geq N_{2}$. Dropping the elements $f_{n}$ with $n<\max \left(N_{1}, N_{2}\right)$ and renumbering, we obtain the desired sequence.

Lemma 8. Let $f$ be a meromorphic function on $\mathbb{C}$, all of whose zeros are multiple, such that $f^{\prime}(z) \neq 1, z \in \mathbb{C}$. Then either

(i) $f$ is rational; or

(ii) there exist nontrivial pairs $\left(a_{n}, c_{n}\right)$ of zeros of $f$ such that $\left|a_{n}-c_{n}\right| \longrightarrow 0$ and a sequence of functions

$$
h_{n}(\zeta)=\frac{f\left(d_{n}+\left(a_{n}-c_{n}\right) \zeta\right)}{a_{n}-c_{n}}
$$

which is not normal on $\Delta$; here $d_{n}=\left(a_{n}+c_{n}\right) / 2$.

Proof. Suppose $f$ is not rational. Then by Lemma $3, f$ has infinite order, so there exist $z_{n} \rightarrow \infty$ and $\varepsilon_{n} \rightarrow 0$ such that

$$
S\left(\Delta\left(z_{n}, \varepsilon_{n}\right), f\right)=\frac{1}{\pi} \iint_{\left|z-z_{n}\right| \leq \varepsilon_{n}}\left[f^{\#}(z)\right]^{2} d x d y \longrightarrow \infty .
$$

Indeed, otherwise there would exist $\varepsilon>0$ and $M>0$ such that

$$
S(\Delta(\zeta, \varepsilon), f) \leq M
$$

for all $\zeta \in \mathbb{C}$. From this follows

$$
S(r)=\frac{1}{\pi} \iint_{|z|<r}\left[f^{\#}(z)\right]^{2} d x d y=O\left(r^{2}\right),
$$

so that (cf. [9, p. 217]) $f$ would have order at most 2 , a contradiction. In particular, there exist $z_{n}^{*} \in \Delta\left(z_{n}, \varepsilon_{n}\right)$ such that $f^{\#}\left(z_{n}^{*}\right) \longrightarrow \infty$. Let $f_{n}(z)=$ $f\left(z+z_{n}^{*}\right)$. Then no subsequence of $\left\{f_{n}\right\}$ is normal at 0 .

Suppose there exists $\delta>0$ such that $f_{n}$ has only a single (multiple) zero $\xi_{n}$ on $\Delta(0, \delta)$. Since no subsequence of $\left\{f_{n}\right\}$ is normal at $0, \xi_{n} \longrightarrow 0$ by Theorem A. Thus, again by Theorem A, $\left\{f_{n}\right\}$ is normal on $\Delta^{\prime}(0, \delta)$. It follows 
from Lemma 7 that there exist $n_{1}<n_{2}<\cdots$ such that for any $a \in \mathbb{C}, f_{n_{k}}-a$ has at most two zeros (counting multiplicity) on $\Delta(0, \delta / 2)$. Thus, for large enough $k$,

$$
S\left(\Delta\left(z_{n_{k}}, \varepsilon_{n_{k}}\right), f\right) \leq S\left(\Delta(0, \delta / 2), f_{n_{k}}\right) \leq 2
$$

which contradicts (3.5).

Thus, for each $\delta>0, f_{n}$ has at least two distinct zeros on $\Delta(0, \delta)$ for sufficiently large $n$. The result now follows immediately from Lemma 5 .

\section{Proof of the Theorem}

Suppose the Theorem is false. Then there exists a sequence $\left\{a_{k}^{*}\right\} \subset D$ with no accumulation point in $D$ and such that $a_{1}^{*} \neq a_{2}^{*}$ and a sequence $\left\{f_{n}\right\} \subset \mathcal{F}$ such that $f_{n} \stackrel{\chi}{\Longrightarrow} f$ on $D \backslash\left\{a_{k}^{*}\right\}$ but no subsequence of $\left\{f_{n}\right\}$ is normal at $a_{1}^{*}$ or $a_{2}^{*}$. We may assume that $a_{1}^{*}=0$ and $D=\Delta$. The argument given in the proof of Lemma 4 shows that $f_{n}^{\prime} \Longrightarrow 1$ on $\Delta \backslash\left\{a_{k}^{*}\right\}$, so $f \not \equiv 0$.

If there exists $\delta>0$ such that $f_{n}$ has only a single (multiple) zero on each $\Delta\left(a_{j}^{*}, \delta\right)(j=1,2)$ for large enough $n$, it follows from Lemma 4 that $f(z)=z-a_{j}^{*}(j=1,2)$ on $\Delta \backslash\left\{a_{k}^{*}\right\}$. Thus $a_{1}^{*}=a_{2}^{*}$, a contradiction.

Therefore, one may suppose that for any $\delta>0, f_{n}$ has at least two distinct zeros on $\Delta(0, \delta)$ for sufficiently large $n$. By Lemma $5, f_{n}$ has a nontrivial pair of zeros in $\Delta(0, \delta)$ for $n$ large enough. Therefore, some subsequence of $\left\{f_{n}\right\}$ (which, as usual, we continue to call $\left\{f_{n}\right\}$ ) has a nontrivial pair of zeros $\left(z_{n}, w_{n}\right)$ such that $\left|z_{n}\right|<1 / n,\left|w_{n}\right|<1 / n$. There exist $\delta_{0}>0$ and $1<s<2$ such that $f_{n} \stackrel{\chi}{\Longrightarrow} f$ on $\Delta^{\prime}\left(0,2 \delta_{0}\right)$ and $f$ does not vanish for $\delta_{0} \leq|z| \leq s \delta_{0}$. For $1 / n<\delta_{0}$, let $\left(a_{n}, c_{n}\right)$ be a nontrivial pair of zeros of $f_{n}$ in $\Delta\left(0, \delta_{0}\right)$ whose distance is minimal. Clearly, $a_{n}-c_{n} \longrightarrow 0$. Set $d_{n}=\left(a_{n}+c_{n}\right) / 2$. Then $d_{n} \in \Delta\left(0, \delta_{0}\right)$; and, passing to a subsequence, we may assume that $d_{n} \longrightarrow a$, so $|a| \leq \delta_{0}$. Since $f$ and $f_{n}$ have no zeros on $\left\{z: \delta_{0} \leq|z| \leq s \delta_{0}\right\}$ if $n$ is large enough, $\left(a_{n}, c_{n}\right)$ is a nontrivial pair of zeros of $f_{n}$ on $\Delta\left(0, s \delta_{0}\right)$ whose distance is minimal.

Set

$$
h_{n}(\zeta)=\frac{f_{n}\left(d_{n}+\left(a_{n}-c_{n}\right) \zeta\right)}{a_{n}-c_{n}} .
$$

Then for each $\zeta \in \mathbb{C}, h_{n}(\zeta)$ is defined if $n$ is sufficiently large. Clearly, all zeros of $h_{n}$ are multiple and $h_{n}^{\prime}(\zeta) \neq 1$. We claim that no subsequence of $\left\{h_{n}\right\}$ is normal on $\mathbb{C}$. Otherwise, taking a subsequence and renumbering, we would have $h_{n} \stackrel{\chi}{\Longrightarrow} h$ on $\mathbb{C}$. Since $\left(a_{n}, c_{n}\right)$ is a nontrivial pair of zeros of $f_{n}$,

$$
h_{n}( \pm 1 / 2)=h_{n}^{\prime}( \pm 1 / 2)=0 \quad \text { and } \quad \sup _{\Delta}\left|h_{n}^{\prime}(z)\right|>1 .
$$


It follows easily that $h^{\prime}(\zeta) \neq 1$ on $\mathbb{C}$ and that $h$ is nonconstant. Since all zeros of $h$ are multiple, Lemma 3 shows that $h$ must be transcendental. It then follows from Lemma 8 that there exist infinitely many nontrivial pairs $\left(\xi_{k}, \eta_{k}\right)$ of zeros of $h$ such that $\xi_{k} \longrightarrow \infty$ and $\xi_{k}-\eta_{k} \longrightarrow 0$, and $z_{k}^{*}$ with

$$
\left|z_{k}^{*}-\frac{\xi_{k}+\eta_{k}}{2}\right|<\left|\xi_{k}-\eta_{k}\right| \quad \text { and } \quad h^{\#}\left(z_{k}^{*}\right) \longrightarrow \infty
$$

Fix $k$ such that $h^{\#}\left(z_{k}^{*}\right) \geq 2$ and $\left|\xi_{k}-\eta_{k}\right|<1$. Then there exist $\xi_{n, k} \longrightarrow \xi_{k}$ and $\eta_{n, k} \longrightarrow \eta_{k}$ such that for $n$ sufficiently large,

$$
h_{n}\left(\xi_{n, k}\right)=h_{n}\left(\eta_{n, k}\right)=0
$$

and

$$
\left|z_{k}^{*}-\left(\xi_{n, k}+\eta_{n, k}\right) / 2\right|<\left|\xi_{n, k}-\eta_{n, k}\right|
$$

Put

$$
\begin{aligned}
& \xi_{n, k}^{*}=d_{n}+\left(a_{n}-c_{n}\right) \xi_{n, k} \\
& \eta_{n, k}^{*}=d_{n}+\left(a_{n}-c_{n}\right) \eta_{n, k} \\
& z_{n, k}^{*}=d_{n}+\left(a_{n}-c_{n}\right) z_{k}^{*} .
\end{aligned}
$$

Then

$$
\begin{aligned}
\left|z_{n, k}^{*}-\frac{\xi_{n, k}^{*}+\eta_{n, k}^{*}}{2}\right| & =\left|a_{n}-c_{n}\right|\left|z_{k}^{*}-\frac{\xi_{n, k}+\eta_{n, k}}{2}\right| \\
& <\left|a_{n}-c_{n}\right|\left|\xi_{n, k}-\eta_{n, k}\right|=\left|\xi_{n, k}^{*}-\eta_{n, k}^{*}\right|,
\end{aligned}
$$

where $\xi_{n, k}^{*} \longrightarrow a, \eta_{n, k}^{*} \longrightarrow a$ and $|a|<s \delta_{0}$; also, for $n$ sufficiently large,

$$
\left|f_{n}^{\prime}\left(z_{n, k}^{*}\right)\right|=\left|h_{n}^{\prime}\left(z_{k}^{*}\right)\right| \geq h_{n}^{\#}\left(z_{k}^{*}\right)>1 .
$$

We conclude that $\left(\xi_{n, k}^{*}, \eta_{n, k}^{*}\right)$ is a nontrivial pair of zeros of $f_{n}$ on $\Delta\left(0, s \delta_{0}\right)$. However,

$$
\left|\xi_{n, k}^{*}-\eta_{n, k}^{*}\right|=\left|a_{n}-c_{n}\right|\left|\xi_{n, k}-\eta_{n, k}\right|<\left|a_{n}-c_{n}\right|
$$

if $n$ is sufficiently large. This contradicts the fact that $\left(a_{n}, c_{n}\right)$ is a nontrivial pair of zeros of $f_{n}$ in $\Delta\left(0, s \delta_{0}\right)$ whose distance is minimal.

Thus no subsequence of $\left\{h_{n}\right\}$ is normal on $\mathbb{C}$. Let $E$ be the set on which $\left\{h_{n}\right\}$ is not normal. Suppose that for each $\zeta \in E$, there is a neighborhood on which $h_{n}$ has only a single (multiple) zero for sufficiently large $n$. Then by Theorem A, $\left\{h_{n}\right\}$ is quasinormal at each point of $E$ and hence on all of $\mathbb{C}$. Let $\zeta_{0} \in E$. Taking a subsequence, we may assume that no subsequence 
of $\left\{h_{n}\right\}$ is normal at $\zeta_{0}$ and that $\left\{h_{n}\right\}$ converges locally spherically uniformly on $\mathbb{C} \backslash E_{0}$, where $E_{0} \subset E$ is a discrete set containing $\zeta_{0}$. By Lemma 4 ,

$$
h_{n} \stackrel{\chi}{\Longrightarrow} \zeta-\zeta_{0} \quad \text { on } \quad \mathbb{C} \backslash E_{0} .
$$

Taking additional subsequences and diagonalizing, we may assume that no subsequence of $\left\{h_{n}\right\}$ is normal at any point of $E_{0}$. We claim that $E_{0}=\left\{\zeta_{0}\right\}$. Indeed, otherwise there exists $\zeta_{1} \in E_{0}, \zeta_{1} \neq \zeta_{0}$; then, as before, it follows from Lemma 4 that

$$
h_{n}(\zeta) \stackrel{\chi}{\Longrightarrow} \zeta-\zeta_{1} \quad \text { on } \quad \mathbb{C} \backslash E_{0},
$$

so that $\zeta_{1}=\zeta_{0}, E_{0}=\left\{\zeta_{0}\right\}$, and

$$
h_{n}(\zeta) \stackrel{\chi}{\Longrightarrow} \zeta-\zeta_{0} \quad \text { on } \quad \mathbb{C} \backslash\left\{\zeta_{0}\right\}
$$

But this contradicts $h_{n}( \pm 1 / 2)=0$. Hence there exists $\zeta_{0} \in E$ such that for each $\delta>0$, there is a subsequence of $\left\{h_{n}\right\}$ (which we continue to call $\left\{h_{n}\right\}$ ) such that each $h_{n}$ has at least two distinct zeros in $\Delta\left(\zeta_{0}, \delta\right)$ for sufficiently large $n$. Then by Lemma 6 , for $n$ sufficiently large, $f_{n}$ has a nontrivial pair of zeros $\left(w_{n, 1}^{*}, w_{n, 2}^{*}\right)$ such that

$$
w_{n, j}^{*} \longrightarrow a(j=1,2) \quad \text { and } \quad\left|w_{n, 1}^{*}-w_{n, 2}^{*}\right|<\left|a_{n}-c_{n}\right| .
$$

This contradicts the fact that $\left(a_{n}, c_{n}\right)$ is a nontrivial pair of zeros of $f_{n}$ in $\Delta\left(0, s \delta_{0}\right)$ whose distance is minimal.

\section{References}

[1] Bergweiler, W. and Langley, J. K.: Multiplicities in Hayman's alternative. J. Aust. Math. Soc. 78 (2005), 37-57.

[2] Chunang, C.-T.: Normal Families of Meromorphic Functions. World Scientific Publishing. River Edge, NJ, 1993.

[3] KU, Y.X.: Un critère de normalité des familles de fonctions méromorphes. Sci. Sinica Special Issue 1 (1979), 267-274 (Chinese).

[4] Nevo, S.: On theorems of Yang and Schwick. Complex Variables Theory Appl. 46 (2001), 315-321.

[5] Nevo, S.: Applications of Zalcman's lemma to $Q_{m}$-normal families. Analysis 21 (2001), 289-325.

[6] Pang, X. C. And Zalcman, L.: Normal families and shared values. Bull. London Math. Soc. 32 (2000), 325-331.

[7] Pang, X. C. And Zalcman, L.: Normal families of meromorphic functions with multiple zeros and poles. Israel J. Math. 136 (2003), 1-9. 
[8] Wang, Y.F. And FAng, M. L.: Picard values and normal families of meromorphic functions with multiple zeros. Acta Math. Sinica (N.S.) 14 (1998), 17-26.

[9] Zalcman, L.: Normal families: new perspectives. Bull. Amer. Math. Soc. (N.S.) 35 (1998), 215-230.

Recibido: 5 de mayo de 2003.

Xuecheng Pang

Department of Mathematics East China Normal University Shanghai 200062, P. R. China

xcpang@math.ecnu.edu.cn

Shahar Nevo

Department of Mathematics

Bar-Ilan University 52900 Ramat-Gan, Israel nevosh@macs.biu.ac.il

Lawrence Zalcman Department of Mathematics

Bar-Ilan University 52900 Ramat-Gan, Israel zalcman@macs.biu.ac.il

This work was done while X. C. Pang held a research position at Bar-Ilan University. He thanks the Mathematics Department of that institution and his collaborators for their warm hospitality. Pang's research was supported by the NNSF of China Approved No. 10271122. The research of all three authors was supported by the German-Israeli Foundation for Scientific Research and Development, G.I.F. Grant No. G-643-117.6/1999. 